\title{
Contribution à l'étude faunistique et écologique des Simulies (Diptera, Simuliidae) du Maroc III. Deux espèces nouvelles du Haut Atlas : Simulium (Simulium) atlasicum n. sp. et Simulium (Simulium) berberum n. sp.1.
}

J. Giudicelli2

A. Bouzidi ${ }^{3}$

Mots clés : Diptera, Simuliidae, nouvelles espèces, hydrobiologie, eaux courantes, Haut Atlas, Maroc.

Deux espèces de Simulies appartenant au groupe de Simulium monticola (Friederichs) sont décrites d'après du matériel récolté dans des ruisseaux froids de haute altitude dans le Haut Atlas central. Simulium atlasicum est connue dans une seule station, à $2100 \mathrm{~m}$ d'altitude. Simulium berberum a été récoltée dans cinq stations entre 2300 et $3200 \mathrm{~m}$ d'al titude.

Contribution to the faunistic and ecological study of the blackflies (Diptera, Simuliidae) of Morocco.

III. Two new species from High Atlas : Simulium (Simulium) allasictum n. sp. and Simulitum (Simulium) berberum n. sp.

Keywords: Diptera, Simuliidae, new species, hydrobiology, running waters, high Atlas, Morocco.

Two new species of Simulium (subgenus Simulium) belonging to the monticola group are described from the central High Atlas (Morocco). Both are high altitude and cool adapted blackflies. Simulium atlasicum was found at one site, a cold spring fed stream $(2100 \mathrm{~m})$. Simulium berberum has been collected from five sites between 2300 and $3200 \mathrm{~m}$.

\section{Introduction}

Simulium monticola Friederichs et les espèces apparentées constituent, en Europe occidentale, un petit ensemble d'espèces sténothermes d'eau froide dont la taxonomie est restée confuse jusqu'à une époque récente.

Simulium monticola Friederichs a été décrite sur du matériel provenant du massif du Harz, en Allemagne (Friederichs 1922).

Doby \& Rault (1960), étudiant des prélèvements provenant de diverses régions de France, ont mentionné pour la première fois l'existence en Europe d'un groupe monticola comprenant Simulium

1. Travail réalisé clans le cadre de l'Action de Recherche Intégree Franco-Marocaine AI 82.T24.

2. Laboratoire de Biologie Animale + Ecologie, Facul té des Sciences de Saint-Jeróme, 13397 Marseille Cédex 13.

3. Laboratoire d'Hydrobiologie. Faculté des Sciences, BP S 15, Marrakech, Maroc. monticola Fried., et une espèce inedite, S. dorieri Doby et Rault.

En 1961, Knoz a reconnu, en Tchécoslovaquie, Odagmia monticola (Fried.) et deux espèces inédites, $O$. rheophila $\mathrm{Knoz}$ et $O$. maxima Knoz, étroitement apparentées à la première.

H. Zwick (1974), puis H. Zwick \& Crosskey (1980), grâce à une analyse précise des différents types, ont établi plusieurs synonymies et ont mis de l'ordre dans ce complexe d'espèces affines. Actuellement le groupe monticola, que Crosskey (1987) rat tache au groupe variegatum, est représenté, en Europe occidentale, par:

- S. argyreatum Meigen $=S$, rheophilum (Knoz), S. monticola auct. nec Friederichs

- $S$. monticola Friederichs $=S$. dorieri Doby et Rault

- S. maximum (Knoz)

- S. sicanum (Rivosecchi) ${ }^{4}$.

4. Crosskey (1987) a mis en synonymie $S$. sicanum avec $S$. monticola. 
Les trois premières ont une large distribution; $S$. sicanum est cantonnée en Calabre et en Sicile (Rivosecchi 1966).

Le groupe monticola est représenté au ProcheOrient par $S$. niha, espèce décrite du Liban par Giudicelli \& Dia (1986). Il existe en Afrique du Nord, signalé au Maroc par Bailly-Choumara \& Beaucournu-Saguer (1981).

A l'occasion d'études hydrobiologiques sur les cours d'eau des massifs du Haut-Atlas marocain, nous avons reconnu deux espèces inédites appartenant à ce groupe; nous en donnons ici les descriptions.

\section{Description de Simulium atlasicum n. sp.}

\subsection{Matériel examiné}

L'holotype et les paratypes or, l'allotype sont des imagos ex-nympha. Tous les exemplaires (nymphes et larves) ont été récoltés dans une seule localité du Haut-Atlas central, près du village d'Aguelmous $\left(31^{\circ} 17^{\prime} \mathrm{N}, 7^{\circ} 25^{\prime} \mathrm{W}\right)$.

Holotype $\sigma$ (monté au baume) : le 28.IV.1985 ; allotype $Q$ (monté au baume) : le $28 . I V .1985$; paratypes : 40,1 \%, 6 nymphes, 3 larves, 28.IV.1985; 2 o, 1 o, 2 nymphes, 5 larves, 27.IV.1987: 3 larves, le 20.IV.1982: 4 \%, 4 \&, 12 nymphes, 4 larves: le 31 .V. 1988.

L'holotype $\sigma$, I'allotype $Q$ et des paratypes sont déposés dans la collection de J. Giudicelli (Marseille) ; plusieurs paratypes sont déposés dans la collection du laboratoire d'Hydrobiologie de la Faculté des Sciences de Marrakech : 2 paratypes ơ et 2 nymphes sont déposés dans la collection de l'Institut Scientifique de Rabat (Maroc).

\subsection{Mâle}

- Tête: Clypeus brun avec reflets argentés. Antennes brunes, les deux premiers articles plus clairs.

- Thorax : Mesonotum brun sombre, couvert de soies dorées couchées; en vue frontale deux taches argentées apparaissent au niveau du tiers antérieur.

- Pattes : Patte I : coxa jaune ; trochanter brun clair ; fémur jaune rembruni à l'extrémité distale ; tibia brun aux tiers distal et proximal, jaune au milieu ; tarses bruns. Patte II : coxa et trochanter bruns; fémur jaune, tiers proximal brun, tiers distal brun clair; tibia brun aux tiers distal et proximal, jaune au milieu; tarses brun noir. Patte III : férnur jaune, excepté le tiers distal brun ; tibia jaune en son milieu, brun aux tiers distal et proximal, le long du bord dorsal de l'article une bande brune réunit les zones brunes apicales ; basitarses jaunâtres, devenant jaune gris dans la moitié distale ; tarses brun noir.

- Genitalia (fig. 1) : Style (longueur : $300-320 \mu \mathrm{m}$ ) trois fois plus long que sa plus grande largeur, nettement courbé vers l'intérieur à partir du milieu : bord externe presque rectiligne ou très faiblement concave ; bord interne concave en son milieu ; extrémité distale arrondie; présence d'une forte épine sub-terminale (longueur : 12-15 $\mu \mathrm{m}$ ), située sur la face dorsale du style et très près du bord latéral ; processus interne très saillant. Rapport entre la longueur du style et la longueur du coxite $=1,70$. Plaque dorsale longue et étroite, trois fois plus longue que large, bords faiblement convexes. Plaque ventrale en $\mathrm{Y}$; corps plus long dans le sens dorsoventral que dans le sens antéro-postérieur ; processus ventral étroit et très long, 2,3 fois plus long que large; deux rangées de 8 à 12 épines sur le bord de la plaque ventrale.

\subsection{Femelle}

Clypeus : brun noir brillant ; occiput brun. Antennes gris brun ; les deux premiers articles et la moitié du troisième jaunâtres.

Pronotum : brun noir brillant, couvert de poils couchés jaune clair brillants.

Genitalia (fig. 2) : Aire médiane du $8^{\mathrm{e}}$ sternite abdominal moyennement sclérifiée, constituée d'une zone centrale déprimée, en forme de trapèze renversé, avec deux expansions latérales de chaque côté, les expansions antérieures étant plus pigmentées et à contours moins nets que les postérieures.

Gonapophyses : bords internes bien écartés l'un de l'autre et ménageant un large espace en forme d'ogive; l'extrémité de chaque gonapophyse se recourbe en un crochet tourné vers l'intérieur et pigmenté en brun; présence de 18 à 20 soies.

Furca : bras très développés et larges, tige étroite. Paraproctes élargis ventralement. Cerques de forme arrondie. Spermathèque noire, sans ornementation de surface.

\subsection{Nymphe (fig. 3)}

Longueur du corps (filaments respiratoires non compris) : 3,7-4,6 mm ; longueur du cocon : 4,9-5,5 mm. Cocon étroit, en forme de pantoufle, étroitement ajusté au corps de la nymphe, formé d'un tissage 

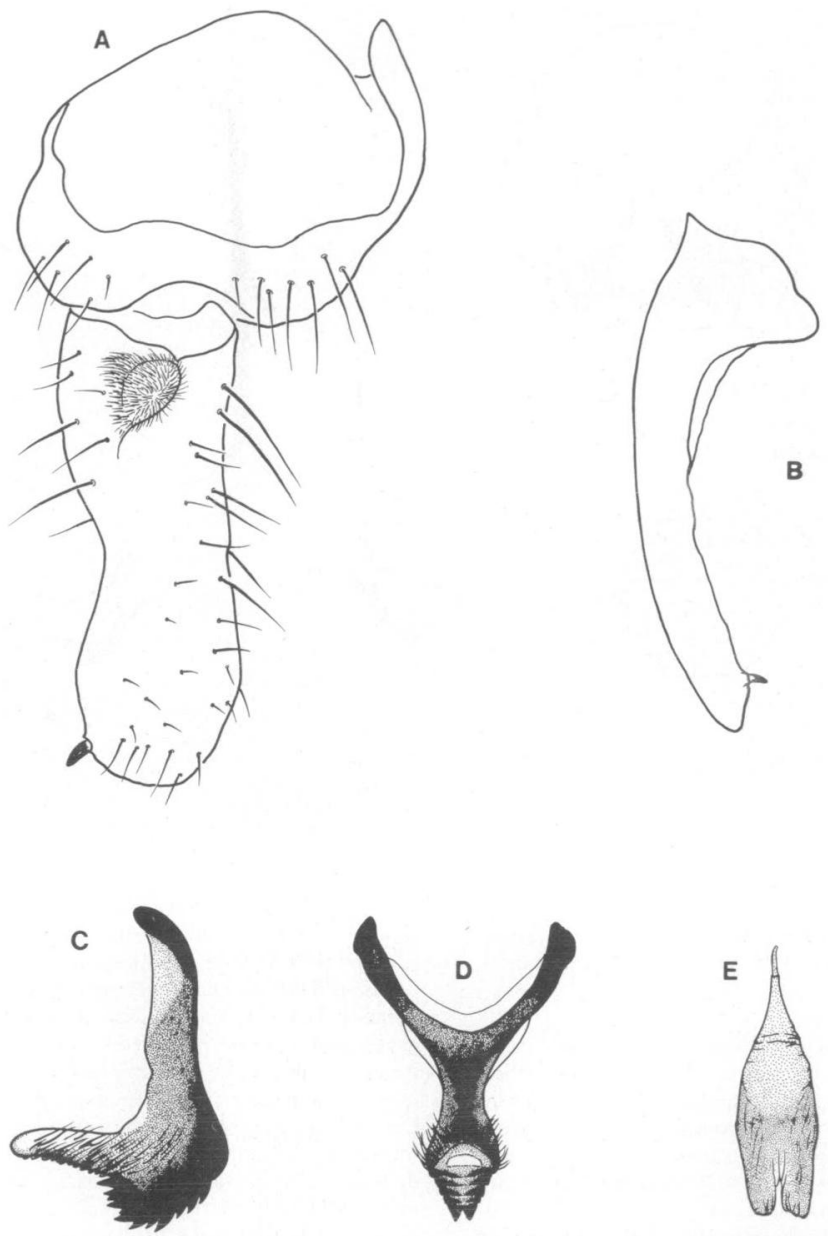

Fig. 1. Simulium atlasicum n. sp. - Mâle.

A : gonopodes en vue dorsale ; $\mathbf{B}$ : gonostyle en vue latèrale; $\mathrm{C}$ : plaque ventrale en vue latérale; $\mathrm{D}:$ plaque ventrale en vue ventrale; $\mathrm{E}$ : plaque dorsale. 


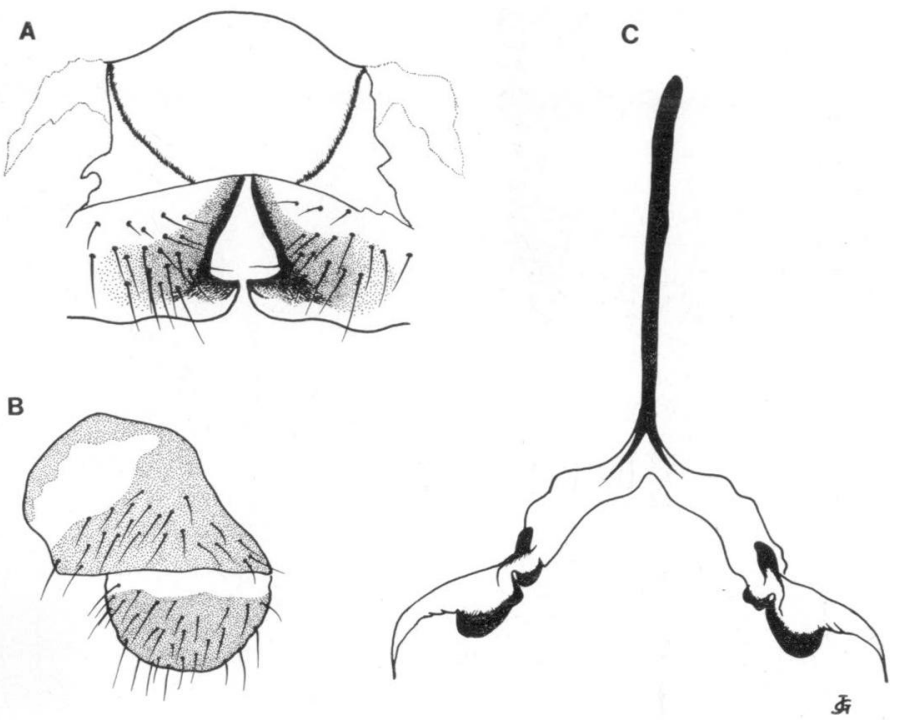

Fig. 2. Simulium atlasicum n. sp. - Femelle.

A : genitalia ; B : paraprocte et cerque; $\mathrm{C}$ : furca.

serré ; bord antérieur renforcé par un bourrelet épais ; les extrémités du bord antérieur se rejoignent et fusionnent sous la face ventrale du corps de la nymphe (fig. 5).

La tète et la partie antérieure du thorax sont dégagées du cocon ; leur tégument est recouvert de tubercules ronds et aplatis (diamètre 12-20 $\mu \mathrm{m}$ ), regroupés en amas irréguliers donnant un aspect granuleux à la partie antérieure du thorax. Sclérite céphalique formant du côte vent ral un angle aigu (en vue de profil) ; surface du sclérite couverte de tubercules denses; de chaque côté du sclérite présence de 3 soies latérales très ramifiées (8-10 filaments secondaires), de 95 à $125 \mu \mathrm{m}$ de long. Trichomes thoraciques ramifiés ( 6 filaments secondaires), de 80 à 90 $\mu \mathrm{m}$ de long.
- Appareil respiratoire (fig. 3) : Il comprend, de chaque côté, 6 filaments à peu près aussi longs que le corps de la nymphe. Filaments disposés en 3 paires, issus d'un tronc basal très épais formant un renflement dorsal. Le tronc basal est inséré sur une grosse protubérance thoracique couverte de nombreux tubercules. Le diamètre des filaments diminue depuis la paire supérieure jusqu'à l'inférieure. Les deux paires supérieures ne sont pas portées par un pédoncule ; les filaments de ces deux paires sont disposés au départ dans un plan horizontal. Les filaments de la paire inférieure sont portés par un court pédoncule et sont disposés dans un plan vertical. Cinq filaments (les 4 filaments des deux paires supérieures et le filament supérieur de la paire inférieure) forment un angle de $45^{\circ}$ avec le support dans leur partie proximale puis s'étalent sur le support ; 
en vue dorsale le filament supérieur diverge et se courbe vers l'intérieur dans sa moitié distale; les 4 autres filaments (surtout les filaments 4 et 5 ) sont plus courbes vers l'intérieur et s'entrecroisent sur la ligne médiane. Le filament inférieur (filament 6) forme un angle très ouvert avec l'autre filament de la mème paire; il se dirige vers l'arrière du corps, s'écartant ainsi du faisceau constitué par les cinq autres filaments; il s'insinue entre le cocon et le thorax et s'entrecroise avec son homologue sur la face dorsale du thorax.
- Abdomen: De part et d'autre de la ligne médiane on relève l'ornementation tégumentaire suivante :

Dorsalement : Une petite soie latérale et 3 soies fines sur le segment $I ; 3$ soies latérales spiniformes et 4 soies spiniformes plus grandes et très rapprochées sur le segment II ; 4 crochets simples rapprochés sur le segment III ; 4 crochets simples plus éloignés les uns des autres sur le segment IV ; 5 petites soies éloignées les unes des autres sur les segments $\mathrm{V}$ et VI ; quelques écailles près de la ligne médiane
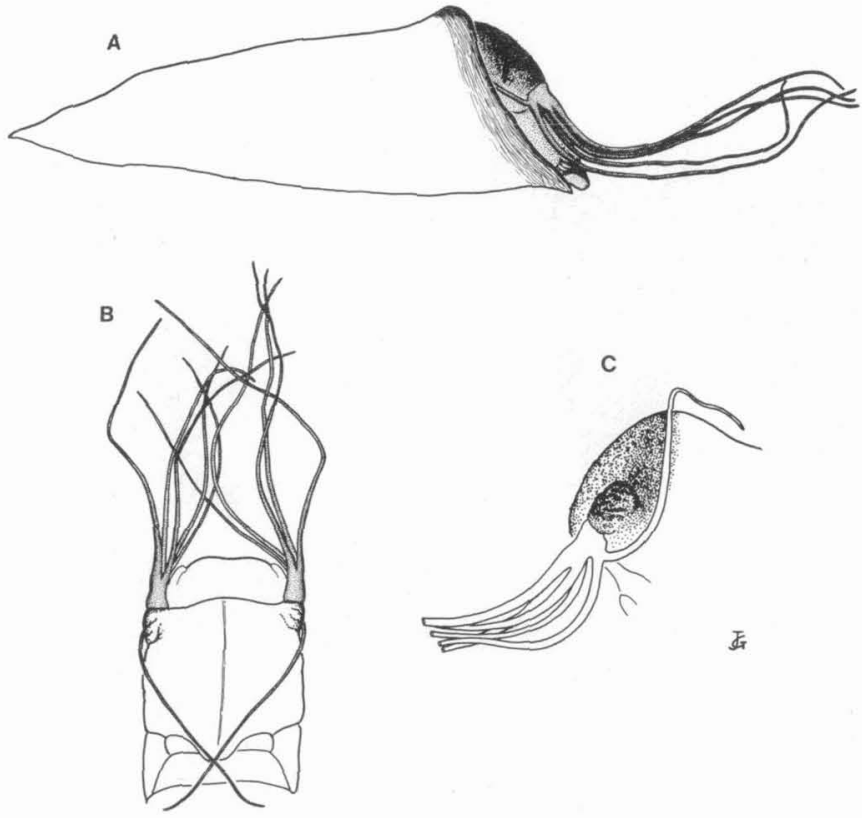

Fig. 3. Simulium atlasicum n. sp. - Nymphe.

A : vue de profil ; $\mathbf{B}$ : extrémité antérieure du corps et filaments respiratoires en vue dorsale ; $\mathbf{C}$ : extrémité antérieure du corps et filaments respiratoires en vue latérale. 

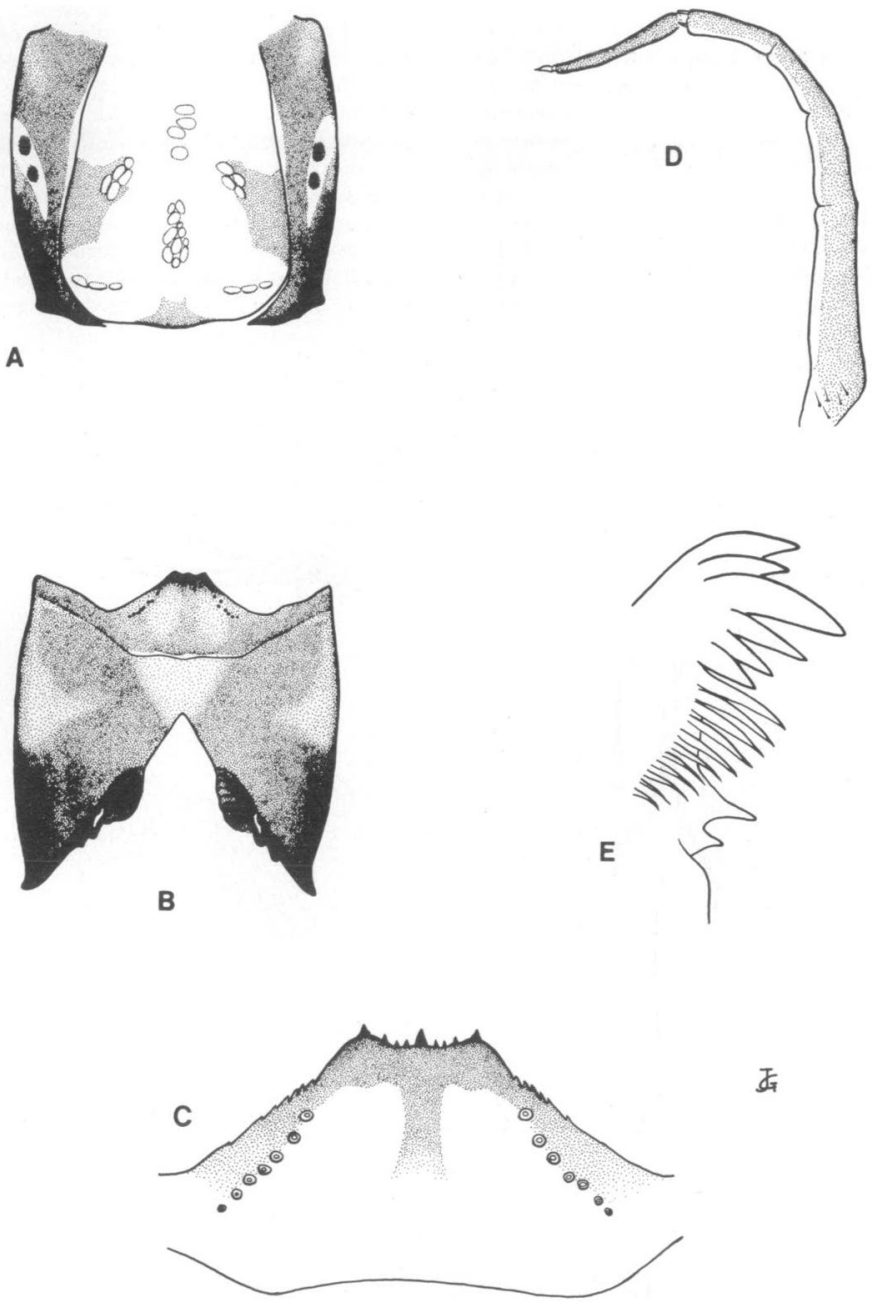

$\mathcal{F}$

Fig. 4. Simulitum ullakicum n. sp. - Lane.

$A$ : capsule céphalique en vue dorsale : $B$ : capsule céphalique en vue ventrale ; $C$ : submentum; $D$ : antenne ; $E$ : extrémité de la mandibule. 
sur le segment VII ; une rangée d'écailles sur le seg. ment VIII ; un tubercule aplati sur le segment IX.

Ventralement : 2 petites soies latérales sur les segments III et IV ; une petite soie latérale et 2 crochets bifides rapprochés sur le segment $\mathrm{V} ; 2$ crochets bifides éloignés l'un de l'autre sur les segments VI et VII.

\subsection{Larve (fig. 4)}

Longueur : 6,9-7.5 mm (larve mûre). Coloration générale grise, capsule céphalique brun clair.

- Fronto-clypeus jaune brun; une tache brune au milieu du bord postérieur ; sur chaque bord latéral une tache brun jaune rejoint les insertions musculaires antéro-latérales.

- Plaques épicraniales brunes, devenant plus claires en avant et latéralement, plus sombres en arrière ; sourcil net ; deux taches oculaires.

- Echancrure ventrale: Sommet triangulaire atteignant la moitié de la distance comprise entre le sommet des apodèmes et le bord inférieur du submentum; bords presque parallèles au niveau des apodèmes.

- Submentum : Bord antérieur garni de 9 dents bien visibles; la dent médiane et les deux dents latérales sont les plus développées ; bords latéraux présentant 5 ou 6 dents; deux rangées latérales de 7 soies simples.

- Antennes : Longueur : $400-430 \mu \mathrm{m} ; 4$ articles, le second étant subdivisé en deux ; les trois premiers articles sont d'un jaune brun très clair, le quatrième est plus sombre.

— Prémandibules : 31 à 33 baguettes falciformes.

- Mandibules : Rangée bt comprenant 10 ou 11 dents; processus $t p$ comprenant une grosse dent aiguë suivie d'une petite dent.

- Abdomen : Absence de papilles ventrales. Branchies rectales portant 7 ou 8 ramifications secondaires. Bras postérieurs du sclérite anal plus longs que les antérieurs ; la cuticule est couverte de petites soies spiniformes entre les bras antérieurs. Disque postérieur comprenant 77 à 85 rangées de 13 à 15 crochets.

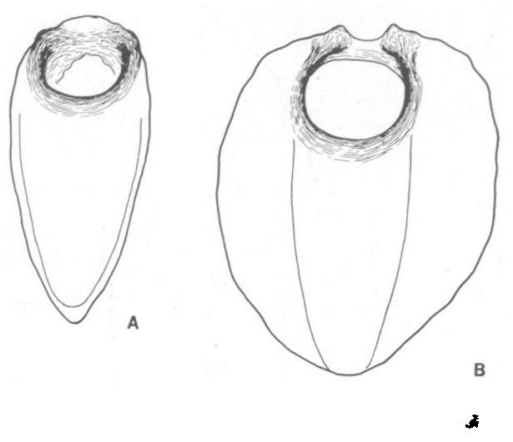

Fig. 5. Cocons nymphaux

A : Simulium atlasicum; B : Simulium berberum.

\section{Description de Simulium berberum n. sp.}

\subsection{Matériel examiné}

L'holotype, les paratypes et l'allotype sont extraits de nymphes múres. Holotype $\sigma$, allotype $\varnothing$ (montés au baume), 4 paratypes $\sigma, 1$ paratype 8,12 nymphes, 14 larves, Maroc : assif n'Ouarzane (réseau superieur de l'oued $\mathrm{N}^{\prime}$ fiss), alt. $3000 \mathrm{~m}, 31^{\circ} 5^{\prime} \mathrm{N} ; 7^{\circ} 58^{\prime} \mathrm{W}, 21$. VII.1985.

3 paratypes o, 19 nymphes, 22 larves, Maroc : même localité, 27.VII. 1985.

3 paratypes $\sigma, 4$ paratypes 9,8 nymphes, 8 larves, Maroc : assif n'Ouarzane, au pied des cascades d'Irhoulidène, alt $2800 \mathrm{~m}, 10$.VIII.1986:17 larves : même station, 10.V.1987.

2 nymphes, Maroc: ruisseau de Tinzart, alt. $2600 \mathrm{~m}$, petit affluent du cours supérieur de l'oued Ourika, $31^{\circ} 7^{\prime} \mathrm{N}$, $7^{\circ} 31^{\prime}$ W, 7.VII. 1984 .

L'holotype, allotype, plusieurs paratypes et paedotypes sont déposés dans la collection de J. Giudicelli (Marseille); plusieurs paratypes sont déposés dans la collection du laboratoire d'Hydrobiologie de la Faculté des Sciences de Marrakech ; 2 paratypes o, 8 nymphes, 4 larves sont déposés dans la collection de l'Institut Scientifique de Rabat.

\subsection{Mâle}

- Tête : Clypeus brun clair avec de longs poils sombres près du bord ventral. Front brun à reflets argentés. Antennes gris brun, les deux premiers articles et le tiers basal du troisième article jaunâtres. 

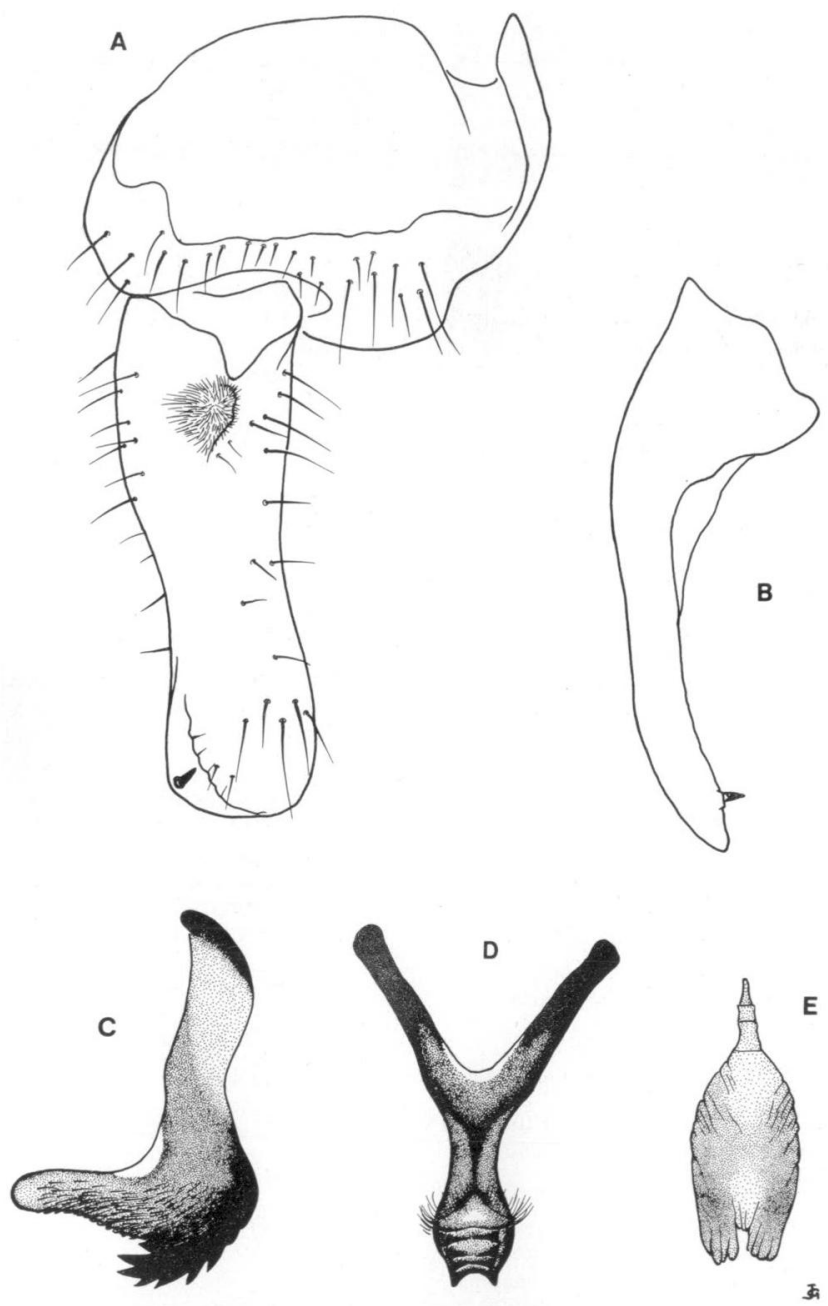

Fig. 6. Simulium berberum n. sp. - Male.

A : gonopode en vue dorsale; B : gonostyle en vue latérale; $\mathbf{C}$; plaque ventrale en vue latérale; $\mathrm{D}$ : plaque ventrale en vue ventrale; $E$ : plaque dorsale. 
- Thorax : Mesonotum brun roux, couvert de soies dorées couchées ; en vue frontale deux taches argentées pales apparaissent sur la partie antérieure.

- Pattes : Patte I : coxa jaune ; trochanter brun clair ; fémur jaune, un peu rembruni à son extrémité distale ; tibia brun aux tiers distal et proximal, jaune au milieu; tarses bruns. Patte II : coxa et trochanter bruns ; fémur jaune avec une tache brune sur le tiers basal du bord superieur ; tibia jaune devenant brun dans le quart distal ; tarse jaune brun. Patte III : coxa et trochanter bruns : fémur jaune, sauf le tiers distal qui est brun; tibia jaune avec une zone brune occupant le tiers distal et descendant jusqu'au milieu du bord supérieur ; basitarse jaune devenant grisâtre dans sa moitié apicale; tarses bruns.

- Genitalia (fig. 6): Style de grande taille (340-355 $\mu \mathrm{m}$ de long) trois fois plus long que sa plus grande largeur, plus étroit dans sa partie antéapicale, caractéristique par sa forme très peu arquée et presque rectiligne, apex paraissant presque tronqué, bords interne et externe légèrement concaves dans la partie médiane, bord externe bombé dans sa partie basale; processus interne du style très saillant; épine sub-terminale longue de 13 à $17 \mu \mathrm{m}$. Rapport entre la longueur du style et celle du coxite : 1.8 à 2 .

Plaque dorsale plutôt large, à bords nettement convexes, 2,6 fois plus longue que large. Plaque ventrale en $\mathrm{Y}$; processus ventral long et conique, 1,5 fois plus long que large; deux rangées de 7.8 épines sur le bord du corps de la plaque ventrale.

\subsection{Femelle}

Clypeus brun clair ; occiput brun roux avec de longues soies claires. Antennes jaunâtres, article basal plus clair.

Pronotum : comme chez le mâle.

Pattes I : Coxa et trochanter bruns ; fémur jaune clair ; tibia jaune clair, rembruni aux deux extrémités ; tarses bruns.

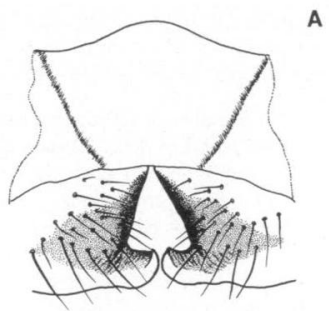

A

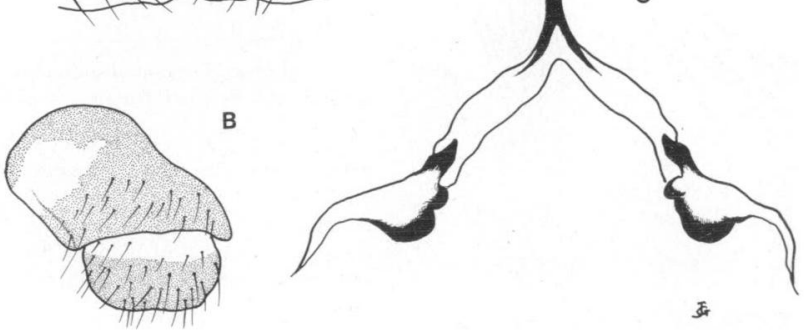

Fig. 7. Simulium berberum n. sp. - Femelle. A : genitalia ; B : paraprocte et cerque ; : furca. 
Pattes II : fémur jaune ; tibia jaune, rembruni à l'apex ; tarses grisâtres.

Pattes III : fémur jaune avec une bande brune à l'apex; tibia jaune, brun à l'apex; basitarse clair dans sa moitié proximale, gris dans sa moitié distale.

Genitalia (fig. 7): Aire médiane sclérifiée du $8^{e}$ stemite abdominal en forme de trapèze, zone centrale déprimée. Gonapophyses bien écartées l'une de l'autre, laissant entre elles un espace en forme de triangle ; extrémité distale recourbée en crochet vers l'intérieur; présence de 22 à 24 soies. Cerques à bord distal presque rectiligne.

\subsection{Nymphe (fig. 8)}

Longueur du corps (filaments respiratoires non compris) : 4,6-5,2 mm.

Tête et partie antérieure dorsale du thorax couvertes de tubercules tégumentaires (diamètre: 19.25 $\mu \mathrm{m}$ ) hémisphériques, finement grenus, isolés ou plus généralement groupés en amas irréguliers.

Sclérite céphalique formant un angle aigu du côté ventral (en vue de profil); de chaque côté présence de 3 soies latérales de 60 à $90 \mu \mathrm{m}$ de long, ramifiées (6 à 8 ramifications).

De chaque côté de la partie antérieure dorsale du thorax se trouve une volumineuse protubérance,

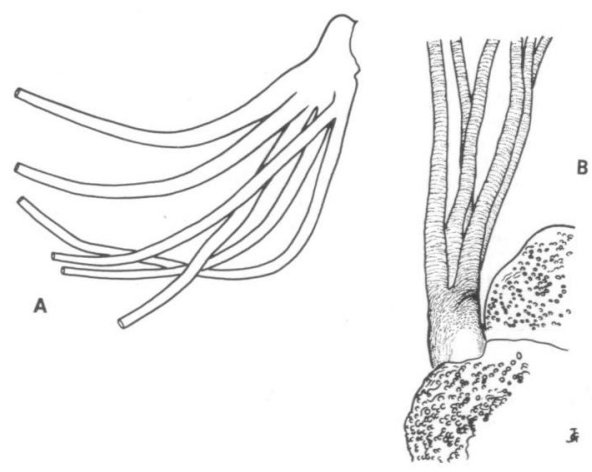

Fig. 8. Simulium berberum n. sp. - Nymphe.

A : filaments respiratoires en vue latérale :

B : filaments respiratoires en vue dorsale. d'aspect ridé et rugueux, couverte de nombreux tubercules tégumentaires serrés. Pas de trichomes thoraciques apparents.

- Appareil respiratoire (fig. 8) : De chaque côté, 6 filaments insérés sur la partie antérieure de la protubérance thoracique. Les filaments sont groupés en faisceau dans leur premier tiers, puis s'incurvent fortement vers le bas et divergent dans leur partie médiane et distale. Les 4 filaments supérieurs ne sont pas disposés en 2 paires portées chacune par un pédoncule mais sont directement insérés sur un gros tronc basal, très court, renflé dorsalement. Les 2 filaments supérieurs sont épais à la base. Le diamètre de la base des 4 filaments supérieurs diminue progressivement depuis le filament 1 jusqu'au filament 4 ; ainsi il est possible de les grouper en 2 paires. Les filaments de chacune des 2 paires supé. rjeures sont disposés dans deux plans inclinés à $45^{\circ}$ par rapport à l'horizontale. Les filaments de la paire inférieure sont portés par un très court pédoncule se détachant du bord vent ral du tronc basal; ils sont situés au départ dans un plan vertical.

En vue latérale, les 6 filaments groupés en faisceau sont orientés au départ vers le support suivant un angle de $45^{\circ}$ environ, puis ils s'incurvent brusquement en s'étalant sur le support.

En vue dorsale, les 6 filaments s'étalent en un faisceau divergent. Les deux filaments des paires supé. rieure et médiane sont divergents au départ; dans la plupart des exemplaires, les filaments 1 et 3 s'écartent du corps de la nymphe ; les deux filaments de chaque paire inférieure se rapprochent sur la ligne médiane et parfois s'entrecroisent avec leur homologue.

- Abdomen : Ornementation tégumentaire identique à celle de $S$. atlasicum.

- Cocon (fig. 5) : Couleur jaune clair, translucide ; texture compacte; forme très aplatie et presque circulaire ; bord antérieur formant un épais bourrelet dont les deux extrémités se rejoignent et se soudent du côté ventral. Le cocon laisse découvertes la tête et la partie antérieure du thorax.

\subsection{Larve (fig. 9)}

Longueur : 8,8-10 mm (larve mûre). Coloration générale gris clair. 


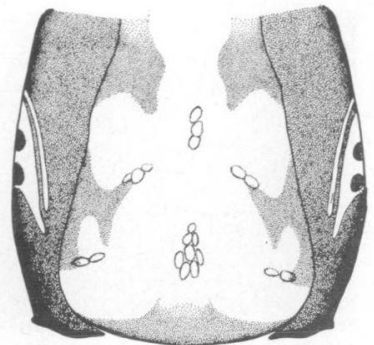

A
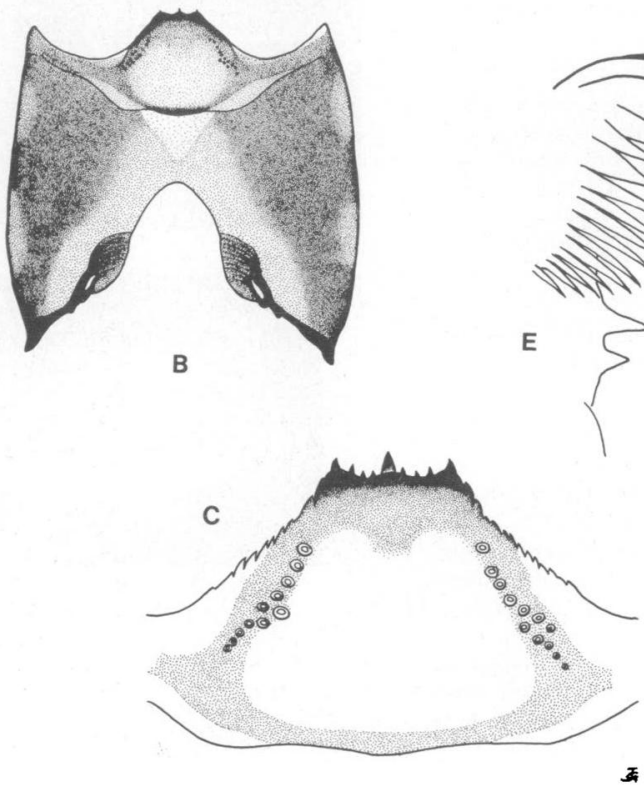

Fig. 9. Simulium berbertum n. sp. - Larve.

A : capsule céphalique en vue dorsale ; B : capsule céphalique en vue ventrale; $C$ : submentrum ; $:$ antenne ; E : extrémité de la mandibule. 
- Fronto-clypeus jaune clair avec une ornementation brune le long du bord postérieur où apparaissent deux taches aux contours nets ; impressions musculaires médianes faiblement pigmentées.

- Plaques épicraniales brunes, devenant jaunâtres près de l'échancrure ventrale ; sourcil net; deux taches oculaires.

- Echancure ventrale large, à sommet arrondi : les bords des apodèmes ne sont pas parallèles.

- Submentum : Neuf dents terminales bien marquées, la dent médiane étant plus saillante que les deux externes; bords latéraux présentant une dizaine de denticulations ; de chaque côté 12 ou 13 soies simples.

- Antennes : Longueur : $480 \mu \mathrm{m}$; dernier article brun, les autres brun clair avec des parties jaunes.

- Prémandibules : 30 à 34 baguettes falciformes.

- Mandibules : Processus $t p$ constitué de 2 dents inégales bien séparées l'une de l'autre.

- Abdomen : Absence de papilles ventrales. Branchies rectales avec 8 à 10 ramifications secondaires sur chaque digitation primaire. Bras antérieurs du sclérite anal plus larges et plus courts que les bras postérieurs; présence d'écailles anales entre les bras antérieurs. Disque postérieur : 85 à 98 rangées de 16 à 19 crochets chacune.

\section{Affinités taxonomiques de Simulium atlasicum et de Simulium berberum}

Les deux espèces marocaines présentent à tous les stades de leur développenent les caractères taxonomiques propres aux espèces du groupe monticola et dont les plus importants apparaissent chez les nymphes.

$S$. allasicum et $S$. berberum ont en commun avec certaines espèces paléarctiques du groupe ( $S$. monticola, S. maximum, S. sicanum, S. niha) un appareil respiratoire nymphal caractéristique avec un tronc basal très épais et dilaté du côté dorsal. Chez ces espèces la disposition des filaments respiratoires apporte de bons critères spécifiques. L'appareil respiratoire de S. atlasicum, avec la disposition particulière du filament le plus ventral, est le plus original.

Le cocon de $S$. berberum, avec sa forme circulaire et aplatie, est bien différent des cocons étroits des autres espèces du groupe monticola.
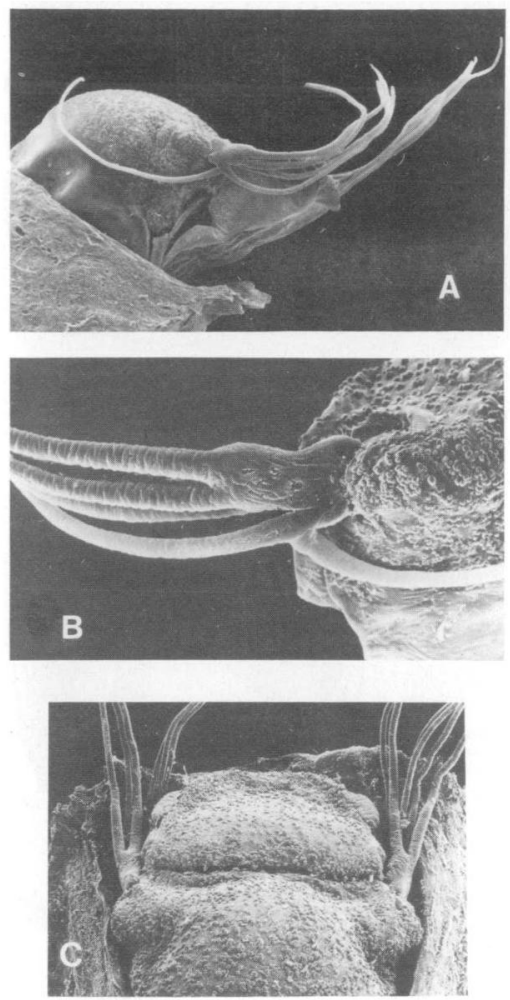

Planche 1. Nymphe de Simulium atlasicum et de Simulium berberum (microscopie électronique à balayage). A : S. atlasicum, extrémité antérieure ; $B$ : S. atlasicum, filaments respiratoires ; $C$ : Simulium berberum, extrémité antérieure. 
Les genitalia des mâles de S. atlasicum et S. berberum ont en commun un caractère remarquable : le processus ventral de la plaque ventrale est etroit et très allongé. Cette particularité ne se trouve que chez $S$. niha. Le rapport entre la longueur $a$ du processus ventral et la hauteur $b$ de la plaque ventrale (voir fig. 10) est de un demi à trois quarts chez les trois espèces méditerranéennes ( $S$. niha $: 0.47, S$. atlasicum $: 0.51, S$. berberum : 0.72 ), de un tiers chez les autres espèces ( $S$. monticola: $0.36, S$. sicanum : 0.33 , S. maximum : 0.34).

A

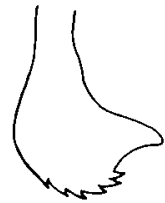

$\mathbf{B}$
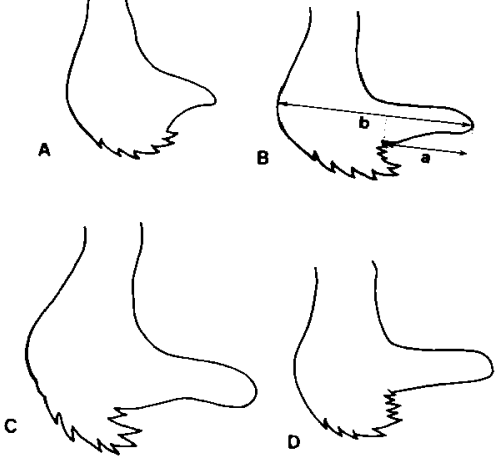

Fig. 10. Plaques ventrales (en vue latérale) de quatre espèces du groupe monticola. A: Simulium monticola (exemplaire de Corse); B : Simulium niha : Simulitum niha ; : Simulitum berbenum ; D : Simulium allasicum.

Les gonapophyses des femelles des deux especes marocaines se terminent en crochet et leurs bords internes sont concaves et très écartés l'un de l'autre. Ces caractères séparent $S$. atlasicum et $S$. berberum des autres espèces européennes du groupe et les rapprochent de $S$. niha.

Ainsi, des affinités étroites semblent lier les deux espèces d'Afrique du Nord et l'espèce du Liban. En plus des particularités communes qui viennent d'être signalées pour les mâles et les femelles, d'autres caractéristiques communes apparaissent chez les nymphes des trois espèces :

- Le sclérite céphalique forme une expansion horizontale du côté ventral ; ainsi, en vue de profil, il fait un angle aigu avec la face ventrale de la tête (cf. fig. 19 et 30 in Giudicelli \& Dia 1986).

- De chaque côté de la partie antérieure dorsale du thorax se trouve une volumineuse protubérance sur laquelle s'insère le tronc basal de l'appareil respiratoire. Cet te disposition existe aussi chez S. humerosum (Rubzov), espèce d'Asie centrale, mais ne figure pas chez les autres espèces du groupe.

- Les tubercules tégumentaires ont une répartition très dense sur le sclérite céphalique et le thorax (caractère observé aussi chez $S$. sicanum). Ils sont moins nombreux chez $S$. monticola (cf. fig. 31 et 32 in Giudicelli \& Dia 1986) et $S$. maximum.

\section{Notes écologiques}

Toutes les espèces du groupe monticola, sont des formes sténothermes. Ce caractère est encore plus marqué chez Simulium berberum et $S$. atlasicum, espèces très alticoles dont les larves et les nymphes vivent dans les secteurs initiaux de cours d'eau prenant naissance dans le plus grand massif du Maroc et d'Afrique du Nord.

Bailly-Choumara \& Beaucournu-Saguez (1981) ont mentionné la présence de Simulies du groupe monticola dans trois stations de la haute Réghaya (entre 1600 et $2300 \mathrm{~m}$ d'altitude) et dans une station de l'oued Ourika (alt. $1450 \mathrm{~m}$ ). Ces Simulies sont représentées dans leurs récoltes de décembre, février et juin, ce qui laisse penser qu'il s'ágissait probablement de larves. Nous avons réalisé de nombreux prélèvements de faune aquatique, sur au moins un cycle annuel avec une fréquence mensuelle, dans les trois stations de la Réghaya citées par Bailly-Choumara et Beaucournu-Saguez. Nous avons trouvé Simulium berbertim uniquement dans la station supérieure (alt. $2300 \mathrm{~m}$ ).

De fait, les représentants du groupe monticola sont rares dans le Haut-Atlas, seul massif du Maroc où ils ont été trouvés. Entre 1984 et 1987 nous avons prospecté l'ensemble des réseaux hydrographiques du massif (74 stations de prélèvements); nous n'avons trouvé ces Simulies que dans six stations, dont cinq sur le versant nord du Haut-Atlas central. 
Simulium berberum est connue entre $2300 \mathrm{~m}$ et $3200 \mathrm{~m}$ d'altitude, dans cinq stations situées dans la partie supérieure de trois cours d'eau qui pren. nent naissance dans le massif du Toubkal.

- Deux stations sur la haute Réghaya

1. au niveau de Sidi Chamharouch (alt. $2300 \mathrm{~m}$ ), (largeur du cours d'eau : 1 à $1,5 \mathrm{~m}$; profondeur : 20 à $50 \mathrm{~cm}$; température de l'eau : $9^{\circ} \mathrm{C}$ en hiver, $15^{\circ} \mathrm{C}$ en été ; conductivitć électrique : $140-160 \mu \mathrm{S} / \mathrm{cm}$ ).

Le peuplement simulidien compte 4 especes : S. variegatum Meig. est l'espèce dominante ; S. toubkal Bouz. et Giud. et S. intermedium Roub. sont constantes mais moins abondantes: $S$. berbertm est rare et peu abondante.

2. Au niveau du refuge Neltner (alt. $3200 \mathrm{~m}$ ), (largeur du cours d'eau : 30 à $70 \mathrm{~cm}$; profondeur : 20 à $30 \mathrm{~cm}$; température de l'eau : 6 à $8^{\circ} \mathrm{C}$ en été : conductivité électrique: $170 \mu \mathrm{S} / \mathrm{cm}$ ). Trois espèces présentes : $S$. berbe . rum dominante avec $S$. toubkal, S. variegattm rare.

- Deux stations sur l'assif n'Ouarzane qui fait partie du réseau supérieur de l'oued N'fiss :

1. A l'aval immédiat des cascades d'Irhoulidène (alt. $2800 \mathrm{~m}$ ), (largeur du cours d'eau : 3,5 à $6 \mathrm{~m}$; profondeur : 20 à $50 \mathrm{~cm}$; température de l'eau : 7,6 à $10^{\circ} \mathrm{C}$ en été ; conductivité électrique : 108 à $115 \mu \mathrm{S} / \mathrm{cm}$ ).

2. Au niveau du refuge de Lépiney (alt. $3000 \mathrm{~m}$ ), (largeur du cours d'eau : $2 \mathrm{~m}$; profondeur : 20 à $30 \mathrm{~cm}$; températu re de l'eau en été : 4 à $5^{\circ} \mathrm{C}$; conductivité : $100 \mu \mathrm{S} / \mathrm{cm}$ ).

— Une station (alt. $2600 \mathrm{~m}$ ) sur l'assif Tinzart au niveau de l'Azib Likemt, affluent du cours supérieur de l'oued Ourika. Espèces compagnes: S. toubkal (abondante) et S. costatum.

Les nymphes apparaissent au début de juillet et se manifestent jusqu'à la fin d'août. Larves e1 nymphes vivent fixées sur les blocs et les pierres dans les courants les plus rapides (70 à $100 \mathrm{~cm} / \mathrm{s}$ ). Les larves matures et les nymphes se groupent en amas, les cocons étant souvent soudés bord à bord les uns aux autres.
Simulium atlasicum a été récoltée dans une seule station sur le versant sud du Haut-Atlas central :

- Cours supćricur de l'assif Imini (alt. $2100 \mathrm{~m}$ ), près du village d'Aguelmous; le ruisseau est presque à sec en été, réduit à un filet d'eau à partir de début de juillet, (largeur du cours d'eau : 50 à $80 \mathrm{~cm}$; profondeur : 10 à $20 \mathrm{~cm}$; température de l'eau : 9,5 à $11^{\circ} \mathrm{C}$ en avril-mai, 12 à $12,5^{\circ} \mathrm{C}$ en juin ; conductivité : 35 à $60 \mu \mathrm{S} / \mathrm{cm}$ ).

Espèces compagnes : Prosimulium cf latimucro, Simulium cf cryophilum, $S$. varieganum.

Les nymphes sont présentes du début avril jusqu'à juin. Larves et nymphes sont toujours isolées, jamais en groupe.

Travaux ciles

Bailly-Choumara (H.) \& Beaucournu-Saguez (F.). 1981. - Contribution à l'étude des Simuljes (Diptera, Simulidae) du Maroc. 2. Le liaut-Atlas. Bult. Inst. Sc. Rabat, 5 : 39-57.

Crosskey (R.W.). 1987. - An annotaled checklist of the world blakflies (Diptera : Simuliidae) : 425-520. In Blackflies. Ecology, poptilation management and annotated world list. Kim \& Mer. rit Ed. Pennsylvania State University Press.

Doby (J.M.) \& Rault (B.). 1960. - Simulies françaises du groupe monticola: Simulium monticola Friederichs, 1920, S, monti. cola var. celtica var. nov, S. dorieri n. sp. (Dipteres Simulii. dés) Bull. Soc Sc. Bretagne, $35: 241-257$.

Friederichs (K.). 1922. - Untersuchungen über Simuliiden. (Teil I1). $Z$. angew. Ent., Berlin, 8:31-92.

Giudicelli (J.) \& Dia (A.). 1986 - Contribution to the knowledge of the Black-ny fauna (Diptera : Simuliidae) of Lebanon, with description of a new species and ecological comments. Aquaiic linsects, $8(3): 123-139$.

Knoz (J.). 1961. - Notes on the species Odagmia monticola (Fried) 1920 (Dipt. Simulitdae) from Czechoslovakia. Zool. Listy. Fol Zool. 10 (2) : $101-116$.

Rivosecchi (L), 1966. - I Simulidi della Calabria. Parasitologia. $8(2): 77-101$.

Zwick (H.) \& Crosskey (R.W.). 1980. - The taxonomy and nomenclature of the Blackflies (Diptera - Simuliidae) described by J.W. Meigen. Aquatic Insects, $2: 225.247$.

Zwick (H.). 1974. - Faunistsch-ökologische und taxonomische Untersuchungen an Similidae (Diplera), unter besonder Beruicksichtigung der Arten des Fulda-Gebietes. Abh. Senckenb. Naturforsch. Ges., 533, 1.116. 